\title{
Renal Pedicle Avulsion in a Hemodynamically Stable Patient: A Case Report
}

\author{
Nabil Louardia, b, Abdessamad Elbahri ${ }^{\mathrm{a}}$, Younes El Harrech ${ }^{\mathrm{a}}$, \\ Mohammed Alami ${ }^{\mathrm{a}}$, Ahmed Ameur ${ }^{\mathrm{a}}$
}

\begin{abstract}
Renal pedicle avulsion in a hemodynamically stable patient is particularly rare. This may be due to relatively well-protected position of the kidneys in the retroperitoneum surrounded by abdominal viscera anteriorly and dense musculature/spine posteriorly. We present a case of hemodynamically stable renal pedicle avulsion injury following closed abdominal trauma.
\end{abstract}

Keywords: Renal pedicle avulsion; Abdominal trauma; Hemodynamically stable

\section{Introduction}

Renal trauma accounts for approximately $3 \%$ of all trauma admissions. Most cases are self-limiting and minor but blunt renal trauma results in a spectrum of injuries ranging from simple contusions to renal pedicle avulsion $[1,2]$.

Renal pedicle avulsion in a hemodynamically stable patient is particularly rare. This may be due to relatively well-protected position of the kidneys in the retroperitoneum surrounded by abdominal viscera anteriorly and dense musculature/spine posteriorly. However, they are vulnerable to acceleration/deceleration injuries as they are only held in space by the renal pelvis and vascular pedicle. Management of renal trauma depends on several factors including hemodynamic stability, grade of renal injury and presence of concomitant injuries [3].

We present a case of hemodynamically stable renal pedicle avulsion injury following closed abdominal trauma.

\section{Case Report}

A 21-year-old male was brought to the Emergency Depart-

Manuscript submitted September 25, 2018, accepted October 4, 2018

aDepartment of Urology, Military Hospital of Instruction Mohammed V, Mohammed V University, Rabat, Morocco

${ }^{b}$ Corresponding Author: Nabil Louardi, Department of Urology, Military Hospital of Instruction Mohammed V, Mohammed V University, Rabat, Morocco. Email: n.louardi@hotmail.fr

doi: https://doi.org/10.14740/wjnu370 ment following a motorcycle traffic accident $2 \mathrm{~h}$ earlier. $\mathrm{He}$ was ejected from the moped after hitting a car.

He complained of left flank pain, thoracic and lumbar back pain, but was otherwise well. The clinical examination had found a conscious patient without hematuria; the blood pressure was $100 / 60 \mathrm{~mm} \mathrm{Hg}$, with tachycardia at 100 beats/ min and polypnique at $30 \mathrm{c} / \mathrm{min}$. Arterial oxygen saturation at room air was $99 \%$. Laboratory tests showed serum hemoglobin at $13.5 \mathrm{~g} / \mathrm{dL}$; he had a normal serum creatinine and urea.

After conditioning, an abdominal CT scan was performed and revealed a rupture of the left renal artery a few millimeters from its origin, with a silent left kidney and a large retroperitoneal hematoma that fuse behind the lesser omental sac without extravasation of the contrast dye (Fig. 1). In addition, the patient had splenic contusion and a complex fracture of the eleventh left rib.

While the patient remained hemodynamically stable and after multidisciplinary consultation (urologists, radiologists and resuscitators), considering the young age of the patient and the location of the lesion, a surgical abstention was decided, with strict and regular monitoring by blood counts twice daily to detect a possible deglobulization. And in this context, the patient received only 2 units of packed red blood cells. A repeat $\mathrm{CT}$ angiogram performed at $\mathrm{J} 3, \mathrm{~J} 7$ and $\mathrm{J} 30$ post-injury revealed a decrease in the retroperitoneal hematoma (Fig. 2).

The patient was managed in an intensive care unit (ICU) and the evolution was marked by a febrile peak at $39^{\circ} \mathrm{C}$, a polypnea and a left thoracic pain. A thoraco-abdominal CT was performed and showed a mean thoracic left chest effusion with bilateral basal atelectasis. The patient benefited from drainage of the effusion by Pleurocath, which brought 1,600 $\mathrm{mL}$ of blood liquid. On the septic plane, we proceeded to ablation of the femoral vein catheter; the culture was in favor of a Staphylococcus aureus (sensitive to tienam and rifampin). After 1 week of treatment, the patient came out of the ICU and was transferred to the urology department.

Our patient will be scheduled in 3 months for a left nephrectomy.

\section{Discussion}

Most traumatic kidney injuries are due to closed trauma caused by traffic or sports accidents. The validation of the classification of these traumas by the American Society of the Surgery of Trauma (ASST) allows a better analysis and management of 


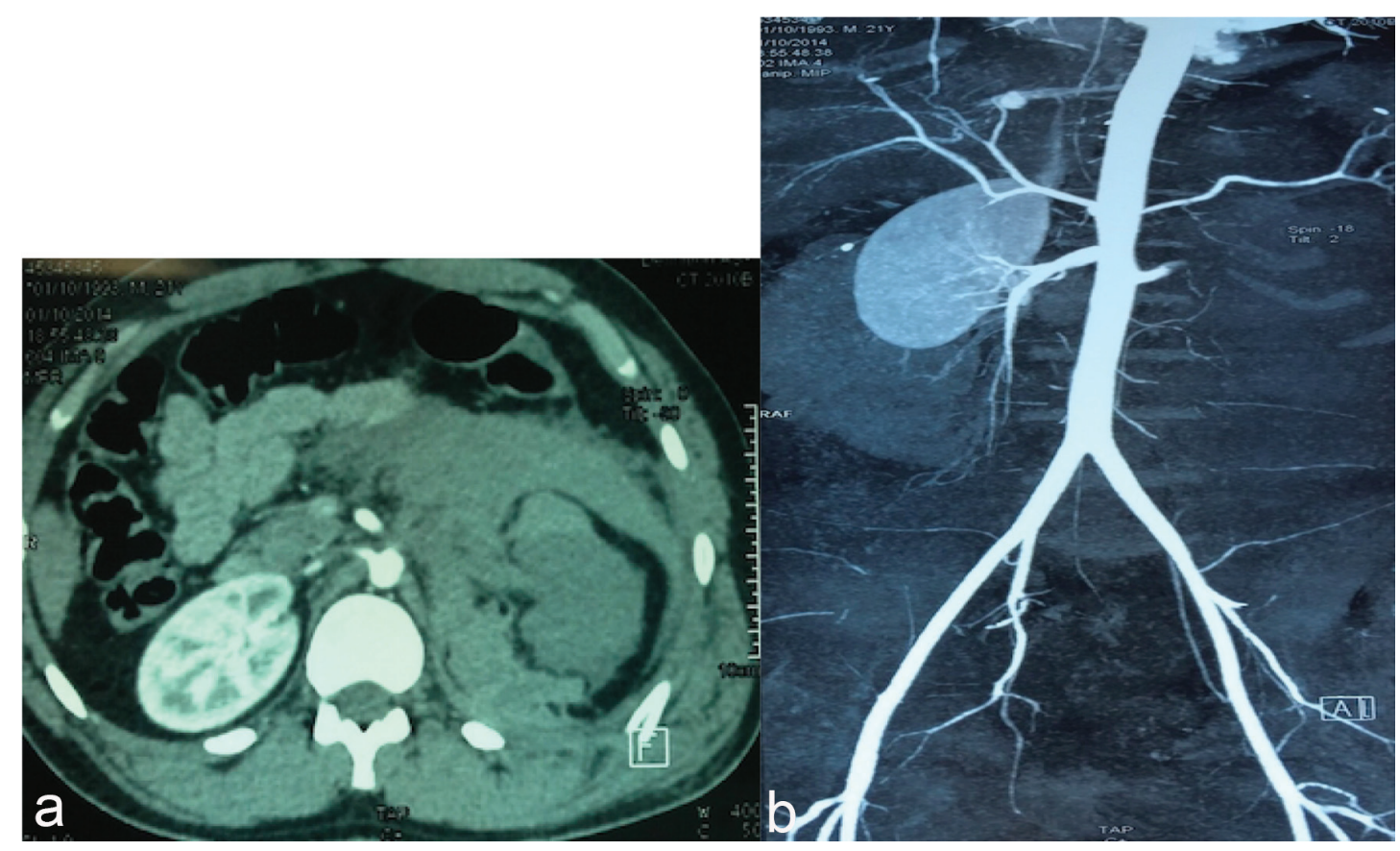

Figure 1. (a) Scan of the day of the trauma; (b) angio-scanner: juxta aortic dissection lesion.
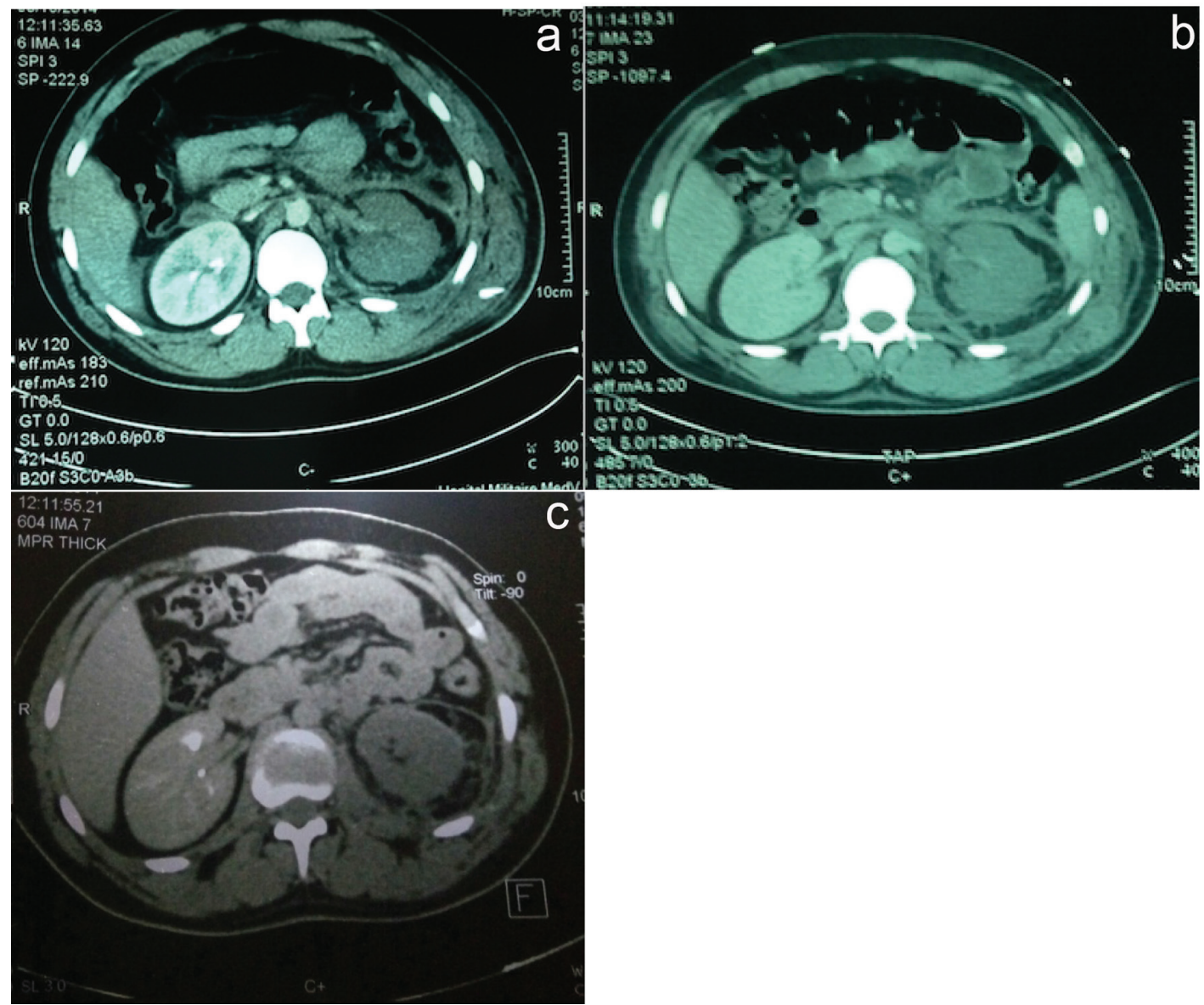

Figure 2. CT angiogram performed at J3 (a), J7 (b) and J30 (c) post-injury. 
these lesions [4]. The imaging indications are now well codified and the spiral CT with contrast injection is the reference examination [5]. For severe trauma (grades 3 - 5), there is still debate between conservative advocates and those advocating surgical intervention [6, 7]. Our case highlights some of the challenges that arise in managing grade 5 renal trauma; the patient was treated conservatively with bed rest, and was admitted to the ICU for overnight observation.

From 1980 to 1985 , most severe kidney injuries (grades 3 - 5) were performed either immediately in some expert centers or delayed (between day 5 and day 10 [8]).

From 1985, several innovative events have qualified this surgical attitude.

The contribution of CT has improved the accuracy of the diagnosis of lesions, and post-traumatic resuscitation has improved considerably.

Grade 5 ASST-classified traumatic renal pedicle lesions are extremely rare and represent $1-4 \%$ of all renal traumas in the series $[9,10]$. The most commonly observed mechanism is abrupt deceleration responsible for the stretching of the pedicle and the rupture of the most fragile vascular tunic: the intima [10]. These lesions are most often found in the context of polytrauma and because of the frequent absence of hematuria, the diagnosis, if it is made, is most often delayed. The absence of hematuria is found in $18-36 \%$ according to the series [10-12].

The treatment of these pedicular lesions may involve: simple observation (attitude retained in our patient) or surgery (total or partial nephrectomy, direct vascular repair, arterial bypass and autotransplantation). One or the other of these methods is the subject of controversy and depends on the delay diagnosis, the existence or not of associated lesions, the experience of the surgeon, the type of lesion, the state of the contralateral kidney and the hemodynamic status of the patient.

In the literature, the results of revascularization are generally disappointing. In a review of 250 patients with stage 5 lesions after closed trauma of the abdomen, Clark et al [13] identified 34 attempts at surgical revascularization, of which eight $(24 \%)$ had a favorable evolution without development of hypertension, but only three patients could be followed after 6 months.

\section{Conclusions}

Conservative treatment in the management of closed kidney trauma in a patient with stable hemodynamic status is now the treatment of choice. The contribution of interventional radiology, endo-urological treatments and the effectiveness of modern resuscitation limit the complications of trauma. Surgical revascularization in renal pedicle injuries is disappointing and revascularization with endovascular stenting is developing with mixed but encouraging results.

\section{Conflicts of Interest}

None.

\section{References}

1. Chatziioannou A, Brountzos E, Primetis E, Malagari K, Sofocleous C, Mourikis D, Kelekis D. Effects of superselective embolization for renal vascular injuries on renal parenchyma and function. Eur J Vasc Endovasc Surg. 2004;28(2):201-206.

2. Dinkel HP, Danuser H, Triller J. Blunt renal trauma: minimally invasive management with microcatheter embolization experience in nine patients. Radiology. 2002;223(3):723-730.

3. Shoobridge JJ, Corcoran NM, Martin KA, Koukounaras J, Royce PL, Bultitude MF. Contemporary management of renal trauma. Rev Urol. 2011;13(2):65-72.

4. Santucci RA, McAninch JW, Safir M, Mario LA, Service S, Segal MR. Validation of the American Association for the Surgery of Trauma organ injury severity scale for the kidney. J Trauma. 2001;50(2):195-200.

5. Descotes JL, Hubert J, Sengel C. [Urology imaging: contribution of medical imaging in renal and ureteral injuries]. Prog Urol. 2003;13(5):1129-1143.

6. Santucci RA, McAninch JM. Grade IV renal injuries: evaluation, treatment, and outcome. World J Surg. 2001;25(12):1565-1572.

7. Schmidlin FR, Rohner S, Hadaya K, Iselin CE, Vermeulen B, Khan H, Farshad M, et al. [The conservative treatment of major kidney injuries]. Ann Urol (Paris). 1997;31(5):246-252.

8. Biserte J, Mazeman E, Lemaitre L, Bertrand P. [Moderately severe kidney injuries. Changes in therapeutic indications]. Chirurgie. 1996;121(5):359-362.

9. el Khader K, Bouchot O, Mhidia A, Guille F, Lobel B, Buzelin JM. [Injuries of the renal pedicle: is renal revascularization justified?]. Prog Urol. 1998;8(6):995-1000.

10. Haas CA, Dinchman KH, Nasrallah PF, Spirnak JP. Traumatic renal artery occlusion: a 15-year review. J Trauma. 1998;45(3):557-561.

11. Carroll PR, McAninch JW, Klosterman P, Greenblatt M. Renovascular trauma: risk assessment, surgical management, and outcome. J Trauma. 1990;30(5):547-552; discussion 553-544.

12. Smith SD, Gardner MJ, Rowe MI. Renal artery occlusion in pediatric blunt abdominal trauma - decreasing the delay from injury to treatment. J Trauma. 1993;35(6):861864.

13. Clark DE, Georgitis JW, Ray FS. Renal arterial injuries caused by blunt trauma. Surgery. 1981;90(1):87-96. 